\title{
Women with fear of childbirth might benefit from having a known midwife during labour
}

\author{
Ingegerd Hildingsson $^{\mathrm{a}, \mathrm{b}, *}$, Annika Karlström ${ }^{\mathrm{b}}$, Christine Rubertsson $^{\mathrm{a}, \mathrm{c}}$, Helen Haines $^{\mathrm{a}, \mathrm{d}}$ \\ a Department of Women's and Children's Health, Uppsala University, Uppsala, Sweden \\ b Department of Nursing, Mid Sweden University, Sundsvall, Sweden \\ ${ }^{\mathrm{c}}$ Department of Health Science, Faculty of Medicine, Lund University, Sweden \\ d Department of Rural Health, University of Melbourne, Wangaratta, Victoria, Australia
}

\section{A R T I C L E I N F O}

\section{Article history:}

Received 28 December 2017

Received in revised form 28 March 2018

Accepted 17 April 2018

\section{Keywords:}

Counseling

Continuity

Fear of childbirth

Pregnancy

Intrapartum care

\begin{abstract}
A B S T R A C T
Aim: Having a known midwife at birth is valued by women across the world, however it is unusual for women with fear of childbirth to have access to this model of care. The aim of this study was to describe the prevalence and factors related to having access to a known midwife for women referred to counseling due to childbirth fear. We also wanted to explore if women's levels of childbirth fear changed over time. Methods: A pilot study of 70 women referred to counseling due to fear of birth in 3 Swedish hospitals, and where the counseling midwife, when possible, also assisted during labour and birth.

Results: $34 \%$ of the women actually had a known midwife during labour and birth. Women who had a known midwife had significantly more counseling visits, they viewed the continuity of care as more important, were more satisfied with the counseling and $29 \%$ reported that their fear disappeared. Fear of birth decreased significantly over time for all women irrespective of whether they were cared for in labour by a known midwife or not.

Conclusions: Although the women in the present study had limited access to a known midwife, the results indicate that having a known midwife whom the women met on several occasions made them more satisfied with the counseling and had a positive effect on their fear. Building a trustful midwife-woman relationship rather than counseling per se could be the key issue when it comes to fear of birth.
\end{abstract}

(C) 2018 Australian College of Midwives. Published by Elsevier Ltd. All rights reserved.

\section{Statement of significance}

\section{Problem or issue}

Having a known midwife during labour is unusual in the Swedish context of care, but all hospital offer counseling due to fear of childbirth.

\section{What is already known}

Women with fear of childbirth prefer continuity with a known midwife during labour.

\begin{abstract}
What this paper adds
Women who had access to a known midwife reported that their fear of childbirth disappeared. It is time to look afresh at the Swedish maternity system and maybe replace the counseling services with continuity models of care for women with childbirth fear and revalue the trustful midwifewoman relationship.
\end{abstract}

\section{Introduction}

Continuity with a known midwife is very important for women with childbirth fear ${ }^{1}$ and is 'best practice' that should be offered to all women regardless of medical risks. ${ }^{2}$ In the Swedish model of health care there is limited continuity from pregnancy to birth. During antenatal care a primary community midwife is assigned to each woman, but if the woman presents with fear of birth, and the midwife notice that the levels of fear is high the woman are often referred to a counseling team, for more specialized care. $^{3}$

\footnotetext{
* Corresponding author at: Department of Women's and Children's Health, Uppsala University, Akademiska sjukhuset ing 95/96, 75385 Uppsala, Sweden. E-mail address: ingegerd.hildingsson@kbh.uu.se (I. Hildingsson).
} 
Counseling for childbirth fear is offered at all Swedish hospitals, ${ }^{4}$ and usually the woman will have 2-3 visits to the counseling midwife. The content of the counseling sessions varies between midwives, but core themes as support, information and childbirth preparation aims to improve the woman's self-confidence in her ability to give birth. ${ }^{3}$

The counseling midwife usually works in collaboration with obstetricians, psychologists or social workers. ${ }^{4}$ It is, however, unusual in most clinics, that the counseling midwife also assists the woman she has met during the counseling visits, when the woman goes in to labour. The reason for this is mainly a belief that women should not be dependent on the counseling midwife, but would have been empowered during the sessions, to be strong enough to manage labour and birth with any midwife.

In addition to the counseling treatment mentioned above, ${ }^{2,3}$ other attempts to treat women with childbirth fear are psychoeducation, ${ }^{5,6}$ cognitive behavioural therapy ${ }^{7}$ and crisis oriented therapy. ${ }^{8}$ In a Swedish pilot study 14 women with severe fear of birth, were offered continuous labour support by one of two specially assigned midwives, in addition to standard counseling. The women met the selected midwives during pregnancy and made a visit to the labour ward to be exposed to the environment and the surveillance technology. The women also had the opportunity to stay in contact with the selected midwives and to schedule subsequent visits. The results showed that women with fear of birth had higher anxiety during labour and were more likely to have induction of labour, compared to a control group of 28 women without fear. ${ }^{9}$ No other differences were found between the groups. Nevertheless, the authors concluded that women with childbirth fear would benefit from continuous support.

The importance of continuity has been widely discussed and the discourse fluctuates between the importance of having the same midwife at all antenatal visits during pregnancy, ${ }^{10}$ and the importance of having a known midwife during labour and birth. $^{11,12}$ Other elements in the discourse of continuity is coherence in philosophy of care (e.g. large teams of 20 midwives). ${ }^{13}$ Continuity with a known midwife builds on a mutual relationship and enables midwives to listen and act upon women's wishes and offer individualized care. ${ }^{12}$ A qualitative interview study with 13 women with fear of birth who received team-midwifery care showed that the relationship with a known midwife was crucial in reducing fear of birth. ${ }^{14}$

Given the inconsistent finding regarding the impact of continuity with a known midwife upon women with a fear of birth, we set out to describe the prevalence and factors related to having access to a known midwife for women referred to counseling due to childbirth fear. We also wanted to explore if women's levels of childbirth fear changed over time.

\section{Material and methods}

We conducted an experimental pilot study where women with fear of birth, when possible, were offered continuity of care during labour and birth from the counseling midwife.

Thirteen counseling midwives working in three hospitals in Sweden, where counseling due to fear of birth was offered, agreed to participate and to provide intrapartum care when they were working at the clinic and a woman with childbirth fear was in labour. The midwives who worked with fear of birth counseling had a long clinical work experienced in the labour ward. However, they were often in charge of the shift as team-coordinators, which means that they were not always available to take care of birthing women and provide continuity of care, rather they have to manage staff and administration on the labour ward. In two of the hospitals there were five midwives providing counseling and in the third hospital three midwives worked with counseling. The midwives spent one day a week on average working with counseling. The referrals counted for 75-100 each year in the three hospitals with a total of 4400 birth all together.

Women referred to midwife-led counseling due to fear of birth with an expected due date between September 12016 and May 31, 2017 were invited to participate. The only inclusion criteria was to understand the Swedish language enough to be able to fill out the questionnaires.

Antenatal midwives referred women to the counseling teams, when women self-reported fear of birth. When women were scheduled for the first counseling visit they received information about the study. They were informed that the counseling visits were designed based on their individual needs and in addition, the counseling midwife, when possible, also assisted during labour and birth. This option had not been possible prior to the project. When a woman agreed to participate through sending in a consent form, the first questionnaire was sent to her home address.

Data was collected by a total of three questionnaires; in mid and late pregnancy and two months after birth. The first questionnaire mainly covered background questions, of which some are presented in the present paper. Two months after birth, questions about the counseling was collected (number of visits, number of midwives met, if the women had had a known midwife at birth, satisfaction with counseling and its effect). The level of fear was assessed in all three questionnaires using FOBS-The Fear of Birth Scale. ${ }^{15,16}$ FOBS consists of two $100 \mathrm{~mm}$ VAS-scales that are summed and averaged to get a score. When filling out the scale study participants are asked to respond to the question "How do you feel right now about the approaching birth?" and are told to place a mark on the two scales, which have the anchor words calm/worried and no fear/strong fear. ${ }^{15}$ The FOBS has been validated in a large Australian study, ${ }^{17}$ in a think aloud study ${ }^{18}$ and in several prospective studies. ${ }^{19,20}$ It has also been used as a screening tool preceding a randomized controlled trial. ${ }^{21}$ The cutoff point of 60 or above is used to categorize women into having fear of birth or not.

We also used the Hospital Anxiety and Depression Scale. ${ }^{22}$ The cut-off points of 8 or more was used to classify women into having anxiety or depressive symptoms. In addition, we also used the Edinburgh Postnatal Depression Scale ${ }^{23}$ with the cut-off point or 13 or more to classify women as having depressive symptoms during pregnancy. ${ }^{24}$ As an indicator of personality traits the Sense of Coherence Scale (SOC) ${ }^{25}$ was used. The 13 items were summed to produce a total score and we thereafter grouped the score into low (-60), moderate (61-75) or high (76-) SOC.

We used descriptive statistics to present the data. We compared women with and without a known midwife at birth, for a variety of selected background variables. For categorical variables we used Chi-square test and for continuous variables Independent t-test. Odds Ratios with a 95\% confidence interval was calculated between women with and without having a known midwife during labour, for the explanatory variables. To test change over time in levels of childbirth fear we used repeated measures analysis of variance. The software SPSS, version 24 , was used in the analysis.

\subsection{Ethical approval}

The study was approved by the regional ethics committee, Dnr 2016/0588.

\section{Results}

In the three hospitals a total of 150 women were referred to counseling, and informed about the study and 77 women (51\%) consented to participate. The main reason for not participating were that women preferred to have a planned caesarean section. Of 
the original sample of 77 women recruited in mid pregnancy, 70 were followed up 2 months after birth.

There were no differences in socio-demographic background between women who completed the follow up questionnaire and those who did not. However, women lost to follow up were more likely to present with a higher FOBS-score in mid pregnancy (mean 85.97) compared to women who returned the follow up questionnaire (mean 70.97, p0.010). They were also more likely to score high on HADS-Anxiety (p0.032).

The sociodemographic and obstetric background of women who returned the follow-up questionnaire is presented in Table 1.

\subsection{Prevalence of having a known midwife at birth}

In total, $15 / 70$ women (21\%) had their primary counseling midwife assisting at birth and of the whole sample 24 women(34.3\%) reported having a known midwife present during some time point of labour and birth. Many women met more than one midwife and 10 women had a known obstetrician present at some point during labour and birth. For further analysis, women were categorized as 'having had a known midwife' and was compared to women who did not have a known midwife during labour and birth.

Table 2 shows that there were no differences between women who had a known midwife at birth and those who did not, with respect to their socio-demographic background or reported mental health in mid pregnancy.
Table 1

Study sample.

\begin{tabular}{lc}
\hline & $\begin{array}{c}\text { Follow up sample } \\
\mathrm{n}=70 \\
\mathrm{n}(\%)\end{array}$ \\
\hline $\begin{array}{l}\text { Age groups } \\
\quad<32\end{array}$ & $29(41.4)$ \\
32 or more & $41(58.6)$ \\
Country of birth & \\
$\quad$ Sweden & $63(90.0)$ \\
Other country & $7(10.0)$ \\
Civil status & \\
Living with partner & $69(98.6)$ \\
Not living with partner & $1(1.4)$ \\
Level of education & \\
Compulsory school/high school & $27(38.6)$ \\
University education & $43(61.4)$ \\
Parity & \\
Primiparas & $18(25.7)$ \\
Multiparas & $52(74.3)$ \\
Previous psychiatric history & \\
Yes & $33(47.1)$ \\
No & $37(52.9)$ \\
\hline
\end{tabular}

Table 2

Comparison of women having a known midwife or not in relation to socio-demographic background, levels of fear and mental health.

\begin{tabular}{|c|c|c|c|}
\hline Data collected in mid pregnancy & $\begin{array}{l}\text { Had a known midwife during labour } \\
\mathrm{n}=24 \\
\mathrm{n}(\%)\end{array}$ & $\begin{array}{l}\text { No known midwife during labour } \\
\mathrm{n}=46 \\
\mathrm{n}(\%)\end{array}$ & p-Value \\
\hline \multicolumn{4}{|l|}{ Age groups } \\
\hline$<2$ years & $10(41.7)$ & $19(41.3)$ & \\
\hline 32 or more & $14(58.3)$ & $27(58.7)$ & 1.000 \\
\hline \multicolumn{4}{|l|}{ Country of birth } \\
\hline Sweden & $21(82.5)$ & $42(91.3)$ & \\
\hline Other country & $3(12.5)$ & $4(8.7)$ & 0.684 \\
\hline \multicolumn{4}{|l|}{ Civil status } \\
\hline Living with partner & $24(100)$ & $45(97.8)$ & not calc \\
\hline Not living with partner & 0 & $1(2.2)$ & \\
\hline \multicolumn{4}{|l|}{ Level of education } \\
\hline Compulsory school/high school & $8(33.3)$ & $19(41.3)$ & \\
\hline University education & $16(66.7)$ & $27(58.7)$ & 0.609 \\
\hline \multicolumn{4}{|l|}{ Parity } \\
\hline Primiparas & $5(20.8)$ & $13(28.3)$ & \\
\hline Multiparas & $19(79.2)$ & $33(71.7)$ & 0.575 \\
\hline \multicolumn{4}{|l|}{ FOBS (mid-pregnancy) } \\
\hline$<60$ & $6(25.0)$ & $11(23.9)$ & \\
\hline$>60$ & $18(75.0)$ & $35(76.1)$ & 1.000 \\
\hline \multicolumn{4}{|l|}{ HADS-anxiety (8 or more) } \\
\hline Yes & $10(41.7)$ & $26(56.5)$ & \\
\hline No & $14(58.3)$ & $20(43.5)$ & 0.315 \\
\hline \multicolumn{4}{|l|}{ HADS-depression ( 8 or more) } \\
\hline Yes & $7(29.2)$ & $11(23.9)$ & \\
\hline No & $17(70.8)$ & $35(76.1)$ & 0.774 \\
\hline \multicolumn{4}{|l|}{ EPDS } \\
\hline$<13$ & $17(70.8)$ & $36(78.3)$ & \\
\hline 13 or more & $7(29.2)$ & $10(21.7)$ & 0.567 \\
\hline Sense of coherence & $8(33.3)$ & $19(41.3)$ & \\
\hline Low & $9(37.5)$ & $13(28.3)$ & \\
\hline Moderate & $7(29.2)$ & $14(30.4)$ & 0.706 \\
\hline High & & & \\
\hline
\end{tabular}


Table 3

Comparison of women having a known midwife or not in relation to care-related variables.

\begin{tabular}{|c|c|c|c|c|}
\hline Data collected 2 months after birth & $\begin{array}{l}\text { Had a known midw } \\
\text { ife during labour } \\
\mathrm{n}=24 \\
\mathrm{n}(\%)\end{array}$ & $\begin{array}{l}\text { No known midwife } \\
\text { during labour } \\
\mathrm{n}=46 \\
\mathrm{n}(\%)\end{array}$ & OR $(95 \% \mathrm{CI})$ & p-Value \\
\hline Number of counseling visits, mean (SD) & $3.20(1.97)$ & $2.06(1.10)$ & $1.8(1.15-2,81)$ & 0.003 \\
\hline \multicolumn{5}{|l|}{ Number of counseling midwives met } \\
\hline One & $21(87.5)$ & $40(87.0)$ & 1.0 Ref. & \\
\hline Two or more & $3(12.5)$ & $3(13.0)$ & $1.9(0.35-10.27)$ & 0.527 \\
\hline \multicolumn{5}{|l|}{ Obstetric consultation due to fear of birth } \\
\hline Yes & $9(37.5)$ & $13(28.3)$ & 1.0 Ref. & \\
\hline No & $15(62.5)$ & $33(71.7)$ & $1.5(0.5-4.3)$ & 0.433 \\
\hline Number of visits to an obstetrician, mean (SD) & $0.37(0.49)$ & $0.36(0.74)$ & $1.01(0.48-2.13)$ & 0.974 \\
\hline \multicolumn{5}{|l|}{ Attitudes towards having a known midwife } \\
\hline Important/very important & $21(87.5)$ & $27(60.0)$ & 1.0 Ref. & \\
\hline Not importnat/rather have an unknown midwife & $3(12.5)$ & $18(40.0)$ & $4.50(1.17-17.30)$ & 0.027 \\
\hline \multicolumn{5}{|l|}{ Satisfaction with midwifery counseling } \\
\hline Satisfied/very satisfied & $23(95.8)$ & $32(71.7)$ & $10.06(1.23-52.03)$ & \\
\hline Not satisfied/mixed feelings & $1(4.2)$ & $13(28.9)$ & 1.0 Ref. & 0.025 \\
\hline \multicolumn{5}{|l|}{ Perceived effect of treatment } \\
\hline My childbirth fear disappeared & $7(29.2)$ & $2(4.5)$ & $16.33(2.19-121.42)$ & 0.006 \\
\hline My childbirth fear decreased & $14(58.3)$ & $26(59.1)$ & $2.51(0.61-110.25)$ & 0.199 \\
\hline My childbirth fear increased & 0 & $2(4.5)$ & & Not calc \\
\hline My childbirth fear was not affected & $3(12.5)$ & $14(31.8)$ & 1.0 Ref. & \\
\hline
\end{tabular}

Table 4

FOBS over time.

\begin{tabular}{|c|c|c|c|c|c|}
\hline & At recruitment & In late pregnancy & $\begin{array}{l}2 \text { months after birth when } \\
\text { looking in retrospect at } \\
\text { the present birth }\end{array}$ & $\begin{array}{l}\text { When thinking about } \\
\text { a future birth }\end{array}$ & p-Value \\
\hline Mean (SD) & $72.18(21.22)$ & $58.68(24.94)$ & $36.75(30.76)$ & $45.79(35.27)$ & 0.000 \\
\hline
\end{tabular}

Table 3 shows a comparison of care-related variables for the two groups of women. The number of counseling visits ranged from 0 to 10 (mean 2.4, Md 2) for the whole sample. There were, however, more counseling visits in the group of women who went on to have a known midwife at birth. The difference was statistically significant. There was no difference in the number of counseling midwives the women met. The majority (87.1\%) met only one counseling midwife, four women (5.7\%) met two midwives and two women (2.9\%) met three or more counseling midwives. Three women reported that they did not receive any counseling appointment with a midwife.

The majority of women did not see an obstetrician due to childbirth fear (68.6\%), but of those 22 who did, 21 were satisfied with the visit. The women who had had a known midwife at birth were significantly more likely to have rated continuity with a known midwife as 'very important' or 'important' during pregnancy.

In all, 55 women were satisfied with the counseling, while 14 women reported mixed or negative feelings and there was a statistically significant difference favouring women with a known midwife. For the majority (58.8\%) the counseling made the fear decrease, for nine women the fear disappeared. For a quarter of the women childbirth fear was not affected by the counseling and two women reported that the fear became worse after having had counseling. When the two groups were compared, it was shown that women who had a known midwife more often reported that their fear disappeared, compared to women who did not have a known midwife.

We finally conducted a one-way repeated measures ANOVA to compare scores of the Fear of Birth Scale (FOBS) at recruitment, in late pregnancy (gestational week 36), and two months after birth. The mean scores are presented in Table 4 . There was a statistically significant change over time, Wilk's Lambda=0,372, F35.59, p0.000, multivariate partial eta square 0.628 . This means that the highest levels of fear was found at recruitment, before counseling, and the lowest two months after birth when thinking in retrospect. Thinking about a future birth made women score higher, but the mean FOBS did not reach the cut-off point of 60 , which is used to classify women into being fearful or not. Similar patterns were found when analyses were done separately for women who had a known midwife and those who did not (Fig. 1).

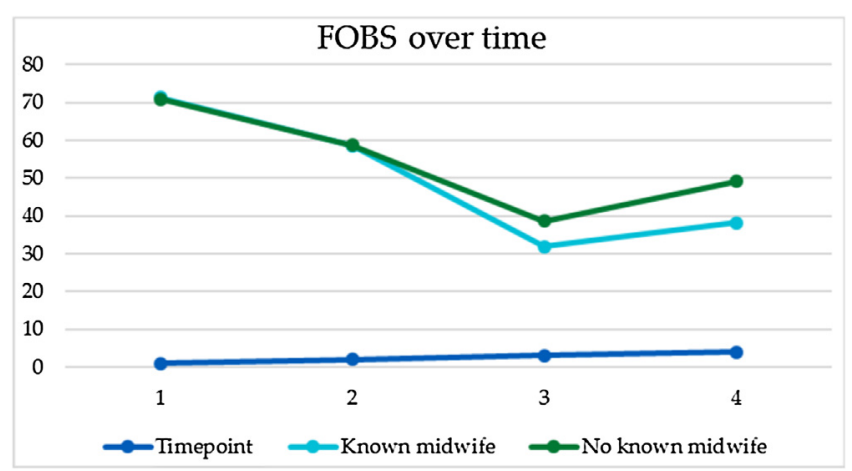

Fig. 1. Mean FOBS over time.

Time point 1: FOBS in mid pregnancy.

Time point 2: FOBS in late pregnancy.

Time point 3: FOBS two months after birth, in retrospect.

Time point 4: FOBS two months after birth, when thinking of a subsequent birth. 


\section{Discussion}

The results of this pilot study indicate that around one third of the women had the opportunity to have a known midwife during labour and birth, but those who did were more likely to report that their levels of fear disappeared. They were also more satisfied with counseling compared to women who did not have a known midwife. The number of counseling visits as well as women's attitude towards continuity were also important. The study also showed that fear of birth decreases over time.

Few women with fear of birth had the opportunity to have a known midwife at birth. The low prevalence could be partly explained by the organization in the labour wards, with many women to care for and where the counseling midwives often are senior with many years of training and often in charge as team coordinators when they worked in the clinic. The midwives in this pilot study did not want to change their working hours and to be "on call' due to private circumstances. Only occasionally, some of the midwives worked beyond their scheduled hours. This situation is very different from the case load models of care where midwives are on call for the women they have provided care for during pregnancy. Most studies have presented that $75-80 \%$ of women in such models have a known midwife at birth. ${ }^{11,26}$ In the Swedish pilot study by Sydsjö et al., ${ }^{9} 12$ out of 14 women with childbirth fear who were assigned a midwife whom they met during pregnancy actually had a known midwife at birth. However, the comparison group with nonfearful women makes any conclusions difficult.

Another reason for the low prevalence of continuity in the present study could be that taking care of women with fear of birth could be emotionally challenging for midwives, as was describe in an interview study of 21 Swedish midwives. ${ }^{27}$ Yet another explanation could be the organization of Swedish maternity care, which is not designed to facilitate continuity. It is known that more than half of pregnant women in Swedish national and regional population-based studies surveys would prefer a known midwife at birth, ${ }^{1,28}$ and women with fear of birth have expressed a stronger need for continuity compared to women without fear.

The barriers for introducing e.g. case load midwifery models of care is mainly due to different healthcare governance structures as Swedish antenatal care are organized through the primary health care sector and is community based or private, while intrapartum care is mainly hospital based. ${ }^{29}$ Midwives usually work in one of these areas, rotation between the two organizations exists but are rare.

We were not able to find any differences in women's background characteristics in relation to having a known midwife during birth or not. This suggest that it was random who actually received continuity. It seems though, that some ingredients of the care played an important role in having a known midwife or not. The number of counseling visits was one such thing. Women who subsequently had a known midwife during birth also had more counseling visits. More counseling visits could have made a reciprocal bonding between the woman and the midwife and it is possible that the midwife identified certain needs in the woman and did her utmost to be there for her. This was also mirrored in the relative grading of the importance of having a known midwife that women presented when they entered the pilot study. The midwife maybe also valued herself as important in the relation. The number of midwives met for counseling or if the woman met an obstetrician was not associated with having a known midwife.

Satisfaction with counseling was another aspect that was strongly represented in women who had a known midwife where $96 \%$ of those who had a known midwife was satisfied with the counseling, versus $72 \%$ of those who did not have a known midwife. Previous population based and randomized controlled trials have reported that between $70-80 \%$ of women exposed to counseling for childbirth fear are satisfied. ${ }^{21,30}$
Finally, when using FOBS, we found that fear of birth decreased over the course of pregnancy, was lowest two months after birth and was slightly increased when the women were thinking of a future birth. This is a quite similar result to what was found in a population based study. ${ }^{16}$ Another interesting finding was the higher proportion (29\%), of women who had a known midwife, reporting that their fear of birth actually disappeared. Most interventions studies regarding childbirth fear, such as psychoeducation, antenatal education and cognitive therapy, have found a decrease in fear over time, as was shown in a meta-analysis of 10 studies, ${ }^{31}$ but we found only one qualitative study that provided continuity of a known midwife for women with fear. ${ }^{14}$ Exactly what factors or circumstances that makes the fear diminish or get less severe is a question yet to be answered.

This study is compromised by the observational design, the fairly small number of participants, and the 'ad hoc' design of the counseling as this naturalistic experiment was incorporated in an already existing provision of care. Nevertheless, the longitudinal design with $90.9 \%$ of the original sample remaining in the study makes the result trustworthy and gives opportunities for further development of models of care for women with fear of childbirth.

\section{Conclusion}

Although the women in the present study had limited access to a known midwife, the results indicate that having a known midwife whom the women met on several occasions made them more satisfied with the counseling and had a positive effect on their fear. Building trustful midwife-woman relationship rather than counseling per se could be the key crucial when it comes to fear of birth.

\section{Implications for practice}

The evidence is mounting that the Swedish system of counseling for fear of childbirth does not have a significant impact on curing women's fear. In the face of this there is a growing body of research pointing towards the positive impact continuity of midwifery care has on reducing women's fear and improving their satisfaction with birth. It is time for a national conversation and a restart of the Swedish maternity system to discuss replacing counseling services with models of care that enable every woman the opportunity to be cared for in labour by a known midwife.

\section{Funding}

This study was funded with grants from the Swedish Research Council.

\section{Conflict of interest declaration}

The authors declare that the article is their original work and it has not been published elsewhere and is not under consideration for publication elsewhere. All authors have seen and approved the final version of the manuscript.

The authors report no conflict of interest.

\section{Ethical approval}

Ethical approval was obtained by the regional ethics committee (no. 2016/0588).

\section{References}

1. Hildingsson I., Haines H, Karlström A, Johansson M. Parents prefer continuity of midwifery care-time to change the Swedish system? A prospective longitudinal cohort study. Sex Reprod Healthc 2016;7:27-32. 
2. Sandall J, Soltani H, Gates S, Shennean A, Devane D. Midwife-led continuity models versus other models of care for childbearing women. Cochrane Database Syst Rev 2015(9)CD004667. doi:http://dx.doi.org/10.1002/14651858CD004667.pub4.

3. SFOG. Svensk Förening för Obstetrik och Gynekologi (Swedish Association of Obstetricians and Gynaecologists). Childbirth fear, ARG rapport nr 7. Linköping: Linköping University; 2017.

4. Larsson B, Karlström A, Rubertsson C, Hildingsson I. Counseling for childbirth fear-a national survey. Sex Reprod Healthc 2016;8:82-7.

5. Rouhe H, Salmela-Aro K, Toivanen E, Tokola M, Halmesmäki E, Ryding E-L. Group psychoeducation with relaxation for severe fear of childbirth improve maternal adjustment and childbirth experience-a randomized controlled trial. J Psychosom Obstet Gynecol 2014;36:1-9.

6. Toohill J, Fenwick J, Gamble J, Creedy D, Buist A, Turkstra E, et al. Effects of a midwife psycho-education intervention to reduce childbirth fear on women's birth outcomes and postpartum psychological wellbeing. BMC Pregnancy Childbirth 2015;30:1-8.

7. Nieminen K, Andersson G, Wijma B, Ryding E-L, Wijma K. Treatment of nulliparous women with severe fear of childbirth via the Internet: a feasibility study. J Psychosom Obstet Gynecol 2016;26:1-7.

8. Nerum H, Halvorsen L, Sörlie T, Öian P. Maternal request for caesarean section due to fear of birth: can it be changed through crisis-oriented counseling? Birth 2006;33:221-8.

9. Sydsjö G, Blomberg M, Palmqvist S, Angerbjörn L, Bladh M, Josefsson A. Effects of continuous midwifery labour support for women with severe fear of childbirth. BMC Pregnancy Childbirth 2015;15:115.

10. Bowers J, Cheyne H, Mould G, Page M. Continuity of care in community midwifery health care. Manag Sci 2015;18:195-204.

11. Haines H, Baker J, Marshall D. Continuity of midwifery care for rural women through caseload group practice: delivering for almost 20 years. Aust J Rural Health 2015;23:339-45.

12. McCourt C, Stevens T, Sandall J, Brodie P. Working with women: developing continuity of care in practice. In: Page L, McCandlish R, editors. The new midwifery: science and sensitivity in practice. 2nd ed. Philadelphia: Churchill Livingstone Elsevier; 2006.

13. Australian College of Midwives. Delivering midwifery continuity of care to Australian women: a handbook for hospitals and health services. 3rd ed. Canberra: Australian College of Midwives; 2017 ISBN 978-1-925358-05-6.

14. Lyberg A, Severinsson E. Fear of childbirth: mothers' experiences of teammidwifery care-a follow up study. J Nurs Manag 2009;18:383-90.

15. Haines H, Pallant J, Karlström A, Hildingsson I. Cross cultural comparison of levels of childbirth related fear in an Australian and Swedish sample. Midwifery 2011;27:560-7.

16. Hildingsson I, Haines H, Karlström A, Nystedt A. Presence and process of Fear of birth during pregnancy-findings from a longitudinal cohort study. Women Birth 2017;30:242-7.
17. Haines H, Pallant J, Toohill J, Creedy D, Gamble J, Hildingsson I, et al. Identifying women who are afraid of giving birth: a comparison of the fear of birth scale with the WDEQ-A in a large Australian cohort. Sex Reprod Healthc 2015;6:20410 .

18. Ternström E, Hildingsson I, Haines H, Rubertsson C. Pregnant women's thought when assessing childbirth related fear on the Fear-of-Birth scale. Women Birth 2016;3:44-9.

19. Ternström E, Hildingsson I, Haines H, Rubertsson C. Higher prevalence of childbirth related fear in foreign born pregnant women-findings from a community sample in Sweden. Midwifery 2015;31:445-50.

20. Haines $H$, Rubertsson C. Pallant J. Hildingsson I. Women's attitudes and beliefs of childbirth and association with birth preference: a comparison of a Swedish and an Australian and Swedish sample in mid-pregnancy. Midwifery 2012;28:850-6.

21. Larsson B, Karlström A, Rubertsson C, Ternström E, Ekdal J, Segebladh B, et al. Birth preference in women undergoing treatment for childbirth fear: a randomised controlled trial. Women Birth 2017;30:460-7.

22. Zigmond AS, Snaith RP. The hospital anxiety and depression scale. Acta Psychiatr Scand 1983;67:361-70.

23. Cox JL, Holden JM, Sagovsky R. Detection of postnatal depression-development of the 10-item Edinburgh postnatal depression scale. $\mathrm{Br} J$ Psychiatry 1987; 150:782-6.

24. Rubertsson C, Börjesson K, Berglund A, Josefsson A, Sydsjö G. The Swedish validation of Edinburgh Postnatal Depression Scale (EPDS) during pregnancy. Nord J Psychiatry 2011;65:414-8.

25. Antonovsky A. The structure and properties of The Sense of Coherence Scale. Soc Sci Med 1993;36:725-33.

26. McLachlan H, Forster D, Davey MA, Farrell T, Flood M, Shafiei T, et al. The effect of primary midwife-led care on women's experience of childbirth: results from the COSMOS randomised controlled trial. BJOG 2016;123:465-74.

27. Salomonsson B, Wijma K, Alehagen S. Swedish midwives' perceptions of fear of childbirth. Midwifery 2010;26:327-37.

28. Hildingsson I, Waldenström U, Rådestad I. Swedish women's interest in homebirth and in-hospital birth center care. Birth 2003;30:11-22.

29. Thomas J, Hildingsson I. Sweden. In: Kennedy, Kodate, editors. Maternity services and policy in an international context. Ireland: Routhledge; 2015 ISBN 978-0-415-73894-1. [chapter 12].

30. Larsson B, Karlström A, Rubertsson C, Hildingsson I. The effects of counseling on fear of childbirth. Acta Obstet Gynecol Scand 2015;94:629-36.

31. MoghaddamHosseini V, Nazarzadeh M, Jahanfar S. Interventions for reducing fear of childbirth: a systematic review and meta-analysis of clinical trials. Women Birth 2017. doi:http://dx.doi.org/10.1016/j.wombi.2017.10.007 pii: S1871-5192(17)30256-1, [Epub ahead of print]. 